\title{
SIMURIS: HIGH-RESOLUTION SOLAR PHYSICS
}

\section{ROBERT J. RUTTEN}

Sterrekundig Instituut, Postbus 80 000, NL-3508 TA Utrecht, The Netherlands

\section{LUC DAMÉ}

Service d'Aeronomie CNRS, BP N ${ }^{\circ} 3$, F-91371 Verrières-le-Buisson Cedex, France

\begin{abstract}
The magnetic fields of the Sun provide the major incentive to do solar physics. The small spatial extent of the magnetically constrained structures and processes in the solar atmosphere provide the major incentive for high resolution solar telescopes. The visibility of the outer solar atmosphere in the ultraviolet and X-ray domains provide the major incentive for solar space telescopes. Cost provides the major incentive to use interferometric techniques. SIMURIS employs short-wave interferometry from space to measure solar structures and processes with high resolution.
\end{abstract}

\section{INTRODUCTION}

SIMURIS ("Solar, Solar System and Stellar Interferometric Mission for Ultrahigh Resolution Imaging and Spectroscopy") is an ESA payload proposal to study the Sun and other nearby objects at high spatial resolution. SIMURIS is described in detail in a recent ESA study report (Coradini et al. 1991) ${ }^{1}$ and in the proceedings of a recent ESA workshop (Damé \& Guyenne 1992).

SIMURIS is intended to be accommodated on Space Station Freedom and to image the Sun simultaneously in the ultraviolet with $0.01^{\prime \prime}$ resolution and in the visible with $0.05^{\prime \prime}$ resolution, employing full-field spectral diagnostics. The SIMURIS payload consists of:

- Solar Ultraviolet Network (SUN). An interferometric aperture-synthesis imager, combining four $20 \mathrm{~cm}$ telescopes in a non-redundant $2 \mathrm{~m}$ baseline array. It achieves coherent beam recombination with continuous co-phasing through active delay-line control, using the large wide-band visible flux for pair-wise fringe acquisition and tracking. Narrow passbands are obtained in three channels with two-dimensional monochromators which are tunable from the ultraviolet to the near-infrared. Full images are obtained by rotational tomography using the ESA Instrument Pointing System. Radio-astronomy techniques are employed for image restoration.

\footnotetext{
${ }^{1}$ Copies available from M. Coradini, ESA, 9-10 Rue Mario Nikis, F-75738 Paris Cedex, France.
} 
- Imaging Fourier Transform Spectrometer (IFTS). A Michelson interferometer, providing spectral line diagnostics for Doppler mapping and polarimetry. It uses a subtractive doubly-dispersing monochromator to limit the detection to only the spectral lines of interest. It accepts two-dimensional fields and has simultaneous outputs in the ultraviolet and the visible.

- Auxiliary instrumentation. Three additional instruments employing plasma diagnostics which differ in characteristic formation temperature (ultraviolet camera, He II $304 \AA$ telescope, multi-layer EUV telescope).

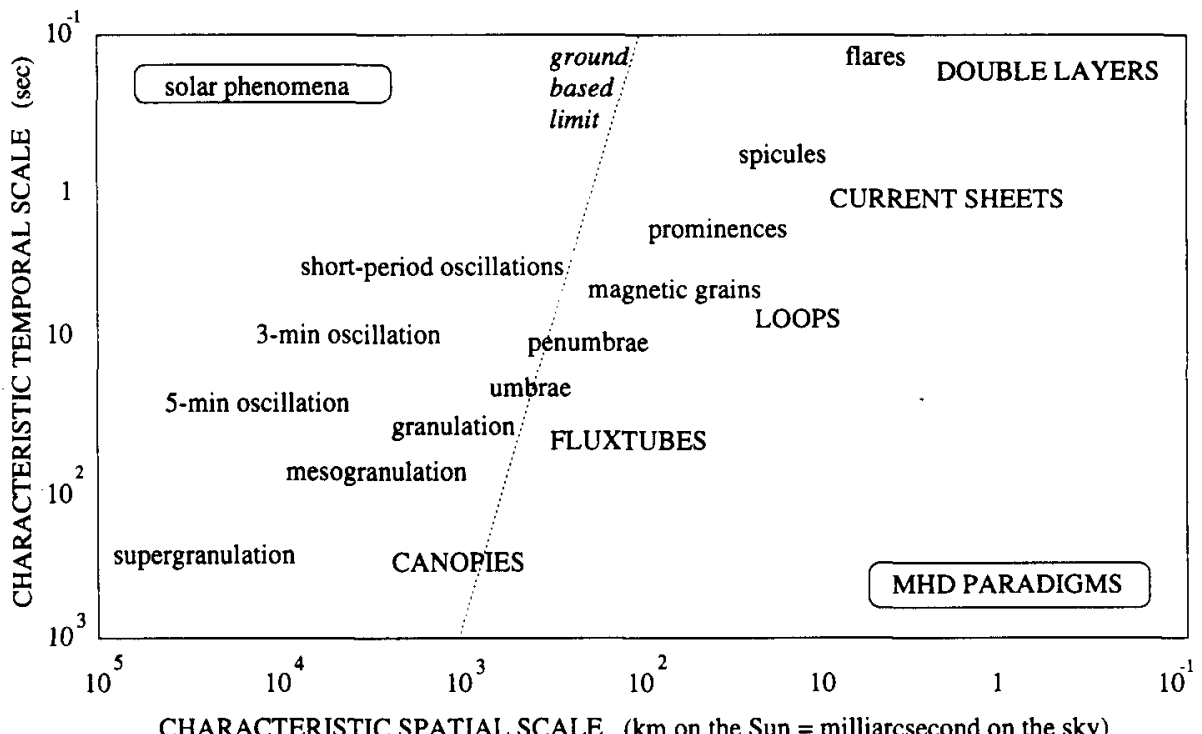

FIGURE I Spacetime characteristics of solar phenomena (small print) and paradigms of magnetohydrodynamics (capitals). The axes specify intrinsic scales, corresponding to desirable resolution. The dotted line is the ground-based resolution limit including new techniques.

\section{SIMURIS SCIENCE}

Figure 1 is a spacetime plot à la Dunn-Harvey-Milkey of which you have seen many incarnations already, including ours (e.g. Rutten \& Cram 1981, Rutten 1990, Coradini et al. 1991, Damé \& Rutten 1992, Rutten 1992). Such plots stress the point that high resolution is the thing to go for in solar physics. The point was valid for SOT-HRSO-OSL, is valid for LEST, and will remain valid in the future. OSL's demise is due to NASA's bad state of affairs (cf. Worden 1992), not to lack of excellent science. The ground-based limit (dotted line) inches slowly towards the right thanks to tilt correction, speckle restoration and adaptive optics; this advance does not diminish the need for high resolution from space but increases it. Numerical simulations, constituting the most productive 
new tool of our era, demand observations commensurate with the diagnostic detail they provide.

The characteristic scales along the axes indicate desirable resolution per phenomenon, expressing the expectation that that resolution suffices to identify the physical nature of that phenomenon. These are intrinsic scales. For example, for understanding prominences one needs to resolve the slender threads of which they probably consist, and the thin interface layer towards the ambient corona (cf. Heinzel \& Vial 1992).

All entries to the right of the dotted line are magnetically constrained. Their explanation necessarily invokes the MHD concepts shown in capitals. The solar atmosphere provides the only location on our sky where these paradigms produce structures and processes that are resolvable.

\section{SIMURIS CONCEPTS}

The characteristic resolutions in Fig. 1 call for apertures in the 1-10 m range. This is modest compared with the desires of galactic and extragalactic astrophysics (see Dainty \& Mattok 1992), but quite long when expressed in ESA accounting units. A filled-aperture solar Space Telescope of $2 \mathrm{~m}$ diameter would require a Hubble-like project. Avoiding $\mathrm{Hubble}$ trouble would require active mirror shape control. The internal solar heating problem would require a Gregorian, of which the alignment would require active configuration control. By that time one has effectively built an active interferometer. Thus, in the $1-10 \mathrm{~m}$ range a filled aperture is not simpler than an interferometer. (In fact, all telescopes are interferometers, but most are passive ones.)

The SUN concept is to use a much cheaper, non-redundant interferometer in which all controls are active. It consists of a telescope array taking snapshot slices for instantaneous high resolution along one dimension, and it uses rotational tomography to construct two-dimensional images. The array is linear because a cross would only halve the rotation time.

The IFTS concept of an array detector in a Michelson spectrometer is the obvious way to go for two-dimensional spectrometry in the ultraviolet. It requires technology development, however, especially to improve the performance of magnesiumfluoride beam splitters at short wavelengths.

The total SIMURIS concept is holistic, aiming to provide multiple diagnostics simultaneously for hot plasmas (X-rays), the optically thin upper atmosphere (ultraviolet) and the optically thick photosphere (visible).

\section{SIMURIS STATUS}

The SIMURIS Study Report (Coradini et al. 1991) is an assessment by a team set up jointly by ESA's Science Directorate and its Directorate of Space Station and Microgravity. SIMURIS is a proposal of interest to both directorates, being a scientific mission which may fruitfully utilize Space Station Freedom facilities. This double interest benefits the project; in particular, ESA has funded a number of detailed design studies ( $c f$. Damé \& Guyenne 1992). There are more to come, including a COLUMBUS precursor flight (Damé et al. 1991). These studies 
make SIMURIS the single space interferometry project that has progressed from Gedankeninstrument ${ }^{2}$ to industrial scrutiny (cf. Dainty \& Mattok 1992).

\section{REFERENCES}

Coradini, M., Damé, L., Foing, B., Haskell, G., Kassing, D., Olthof, H., Mersch, G., Rutten, R. J., Thorne, A. P., Vial, J.-C. 1991, ESA SCI, (91) 7, 1

Dainty, C., Mattok, C. (eds.) 1992, Targets for Space-Based Interferometry, ESA SP-354, ESA Publ. Div., Estec, Noordwijk, in press

Damé, L., Curdt, W., Marsch, E., Hammer, R., Mattig, W., Birkmayer, W., Lutz, R., Rutten, R. J., Braam, B., Visser, H., Tondello, G., Harrison, R., Patchett, B., Delaboudinière, J.-P., Foing, B., Vial, J.-C., Malaise, D., Giner, J. 1991, Solar Ultraviolet Network Spacelab Version (SUNSV), Complementary proposal submitted for the COLUMBUS Precursor Flight Spacelab-2, Service d'Aéronomie du CNRS, Verrières-le-Buisson

Damé, L., Guyenne, T.-D. 1992, Solar Physics and Astrophysics at Interferometric Resolution, ESA SP-344, ESA Publ. Div., ESTEC, Noordwijk

Damé, L., Rutten, R. J. 1992, in L. Damé, T.-D. Guyenne (eds.), Solar Physics and Astrophysics at Interferometric Resolution, ESA SP-344, ESA Publ. Div., ESTEC, Noordwijk, p. 21

Heinzel, P., Vial, J.-C. 1992, in L. Damé, T.-D. Guyenne (eds.), Solar Physics and Astrophysics at Interferometric Resolution, ESA SP-344, ESA Publ. Div., ESTEC, Noordwijk, p. 57

Rutten, R. J. 1990, in J.-O. Stenflo (ed.), Solar Photosphere: Structure, Convection and Magnetic Fields, IAU Symposium 138 (Kiev), Kluwer, Dordrecht, p. 501

Rutten, R. J. 1992, in C. Mattok (ed.), Targets for Space-based Interferometry, ESA SP-354, ESA Publ. Div., ESTEC, Noordwijk, in press

Rutten, R. J., Cram, L. E. 1981, in S. D. Jordan (ed.), The Sun as a Star, CNRS-NASA Monograph Series on Nonthermal Phenomena in Stellar Atmospheres, NASA SP-450, Washington, p. 3

Worden, S. P. 1992, in M. S. Giampapa, J. A. Bookbinder (eds.), Seventh Cambridge Workshop on Cool Stars, Stellar Systems and the Sun, Astron. Soc. Pac. Conf. Series, Vol. 26, p. 599

Zirin, H. 1992, in J. T. Schmelz, J. C. Brown (eds.), The Sun: A Laboratory for Astrophysics, NATO-ASI Series C-373, Kluwer, Dordrecht, p. 379

\footnotetext{
${ }^{2}$ Zirin (1992) defines Gedankeninstruments as: virtual instruments that are non-existent or improbable devices, normally proposed to government agencies or panels of theoreticians.
} 